\title{
Vietato studiare, vietato insegnare
}

di Nina Quarenghi

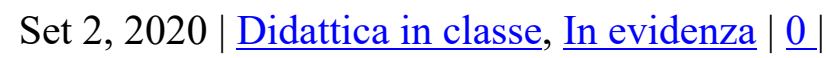

Le norme antiebraiche applicate al mondo della scuola (1938-1943)

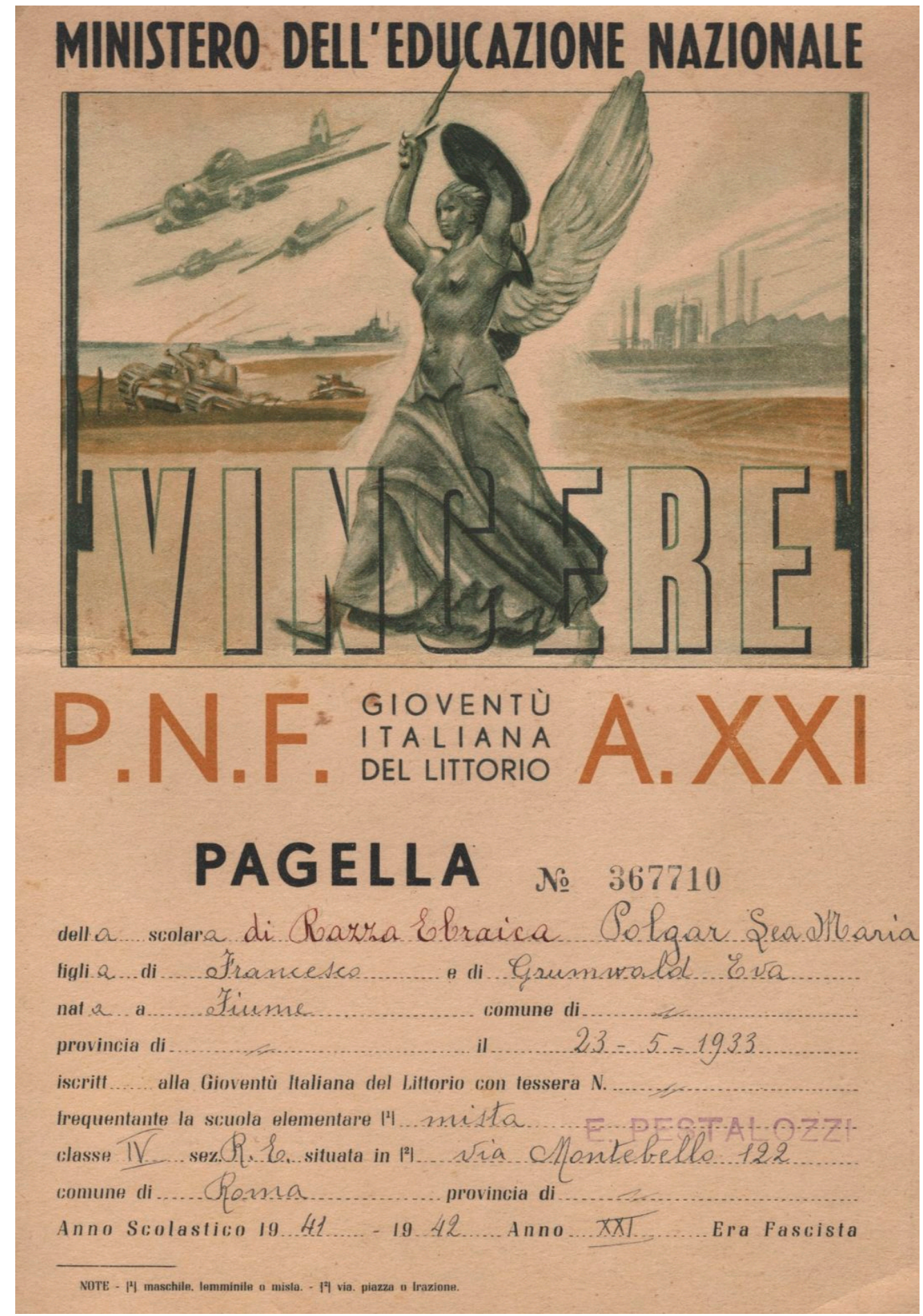

Pagella conservata presso l'archivio privato Lea Polgar 


\begin{abstract}
Dal 1938 al 1943 le leggi razziali, che colpirono la minoranza ebraica in ogni aspetto della vita pubblica, furono applicate con particolare tenacia al mondo della scuola e della cultura. In questo laboratorio didattico scopriremo, attraverso l'analisi di documenti originali e testimonianze orali, come si arrivò a definire questa normativa e cosa significò, per studenti, insegnanti e personale scolastico essere esclusi dalle aule di scuola. L'attività è stata preparata in collaborazione con la Biblioteca «Luigi De Gregori» del Miur che, nel 2019, ha pubblicato il volume, curato da Vincenza Iossa e Manuele Gianfrancesco, Vietato studiare vietato insegnare. Il Ministero dell'educazione nazionale e l'attuazione delle norme antiebraiche 1938-1943, Palombi editori, 2019, pp. 284. Il volume è stato divulgato anche in pdf sul sito Scuola e Memoria: https://www.scuolaememoria.it/site/it/2019/01/25/vietato-studiare-vietato-insegnare/.
\end{abstract}

\title{
L'attività didattica
}

Il laboratorio è pensato per una classe di scuola secondaria, di primo o secondo grado.

Ha la durata di circa due ore:

- 45 minuti: spiegazione dell'argomento attraverso un PowerPoint (SCARICA QUI);

- 1 ora: laboratorio sui documenti (SCARICA QUI) così organizzato:

- 30 minuti: la classe viene divisa in gruppetti di tre-quattro studenti, ciascuno dei quali avrà un documento da analizzare seguendo una scheda-guida (SCARICA QUI);

- 30 minuti: i gruppetti a turno relazionano alla classe i risultati della loro analisi.

\section{Selezione di documenti}

Per la realizzazione dell'attività proponiamo una selezione di documenti di varia natura. Il consiglio è quello di stamparli a colori (e possibilmente plastificarli così da poterli riutilizzare) e, dopo la spiegazione realizzata con l'ausilio del PowerPoint, distribuirli agli studenti che saranno divisi in gruppi di tre-quattro ciascuno.

Ogni gruppo riceverà un documento, accompagnato da una scheda di analisi uguale per tutti. Dopo mezz'ora dedicata al lavoro di analisi, si avranno a disposizione cinque minuti per relazionare alla classe il risultato della propria breve indagine. Si suggerisce di accompagnare quest'ultima fase con la proiezione dei documenti proposti: in questo modo l'intera classe potrà conoscere l'intero dossier e seguire la ricostruzione storica proposta dai compagni.

\section{Documenti:}




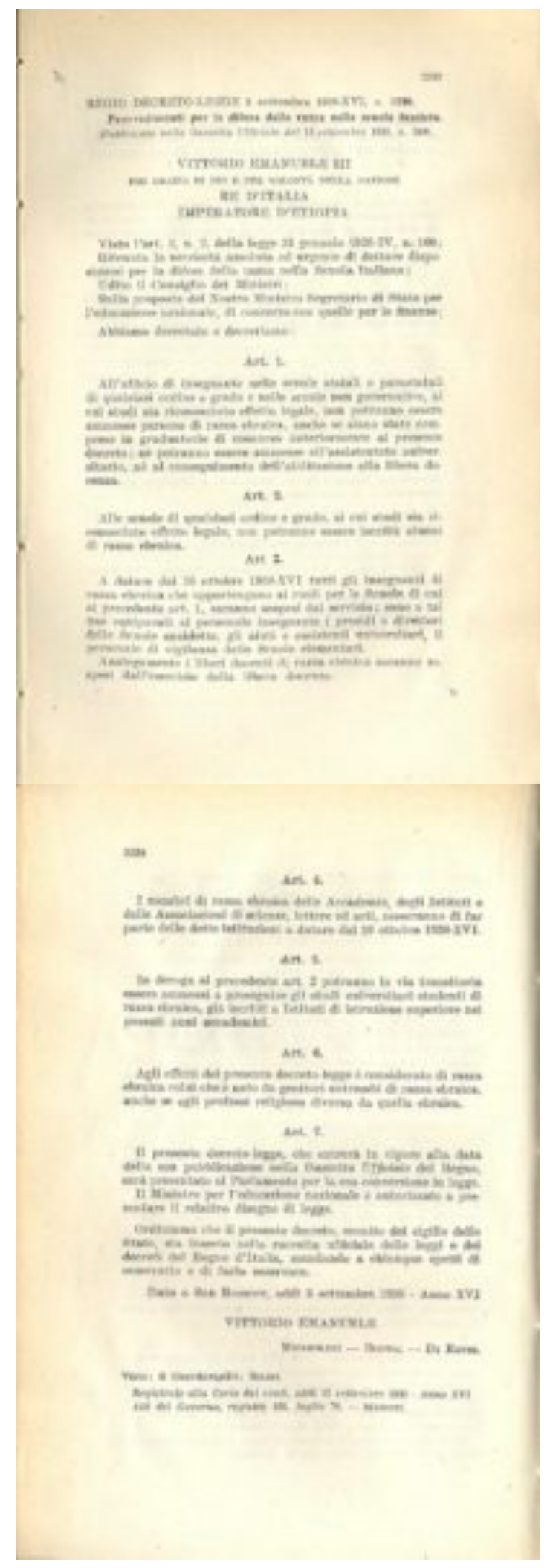

1. (fronte/retro): Regio decreto-legge, 5 settembre 1938-XVI, n. 1390. Ministero dell'Educazione Nazionale, Bollettino ufficiale n. 39, in Vietato studiare, vietato insegnare. Il Ministero dell'Educazione Nazionale e l'attuazione delle norme antiebraiche, , pp. 41-42. 


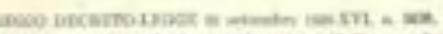

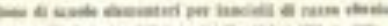

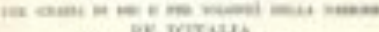

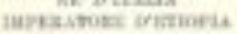

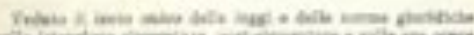

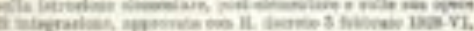

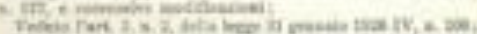

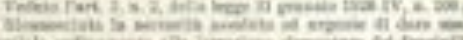

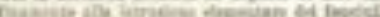

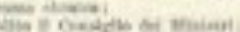

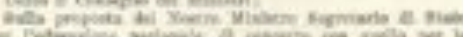
-

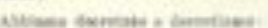

A+4. 1

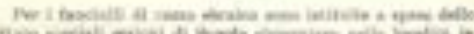

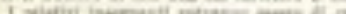

an: 7

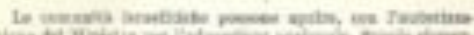

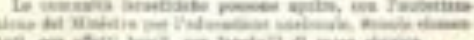

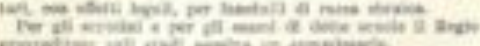

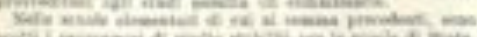

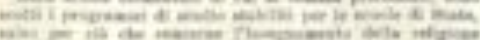

anin

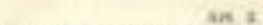

an $\mathrm{x}$

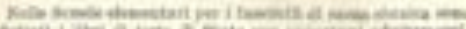

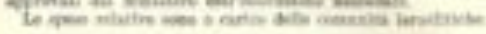

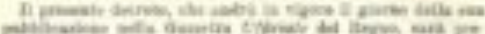

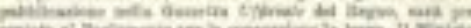

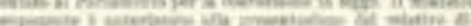
ment aing

mint

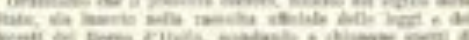

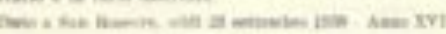
Toxmas exosricis

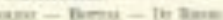

for

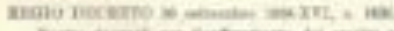

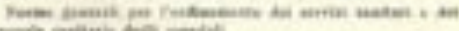

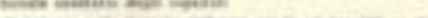

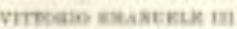

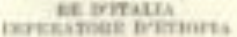

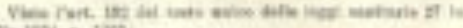

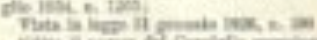

mean ox ous

2. (fronte/retro): Regio decreto-legge, 23 settembre 1938-XVI, n. 1630. Ministero dell'Educazione Nazionale, Bollettino ufficiale n. 45, in Vietato studiare, vietato insegnare. Il Ministero dell'Educazione Nazionale e l'attuazione delle norme antiebraiche, , p. 53. 


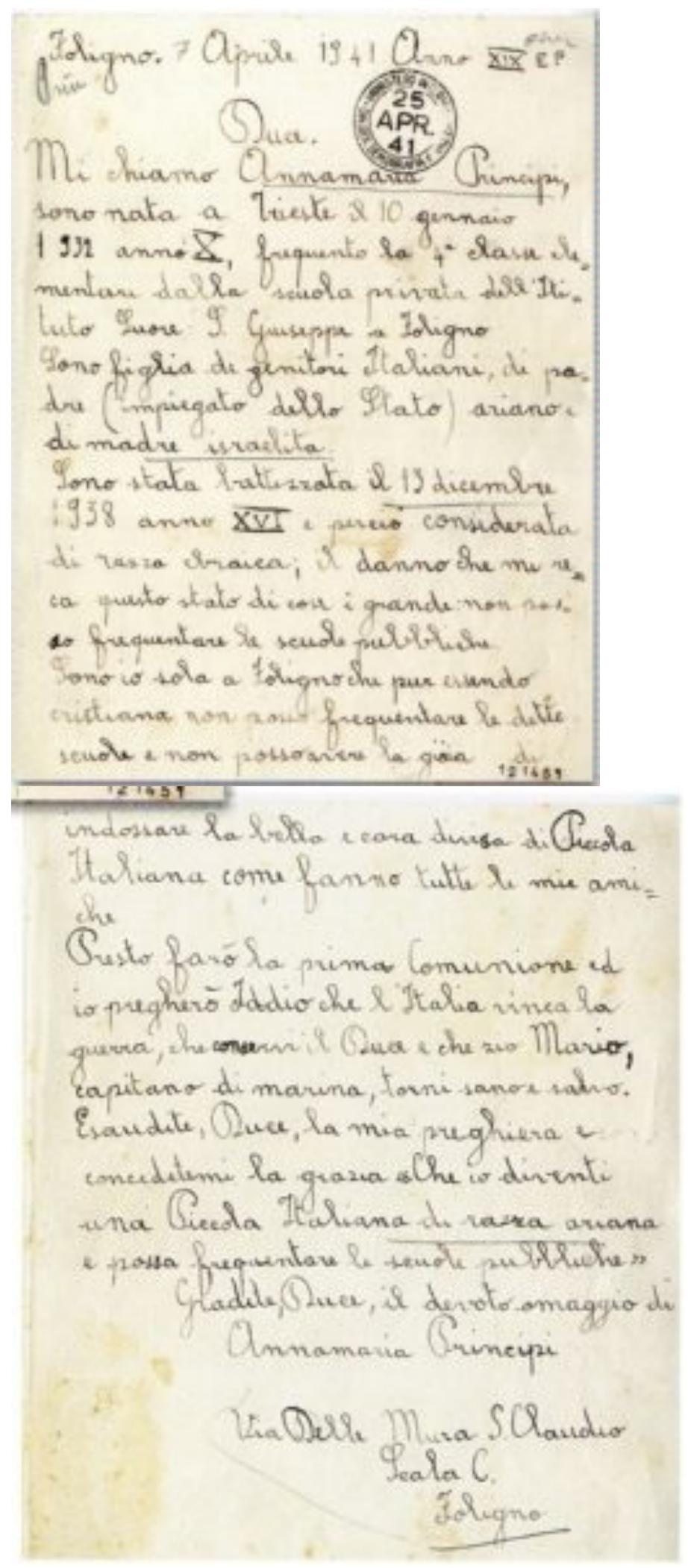

3. (fronte/retro): Lettera di una bambina al duce. Archivio centrale dello Stato, Roma, in S. Berger e M. Pezzetti (a cura di), La storia, Gangemi Editore, 2017, p. 137. 


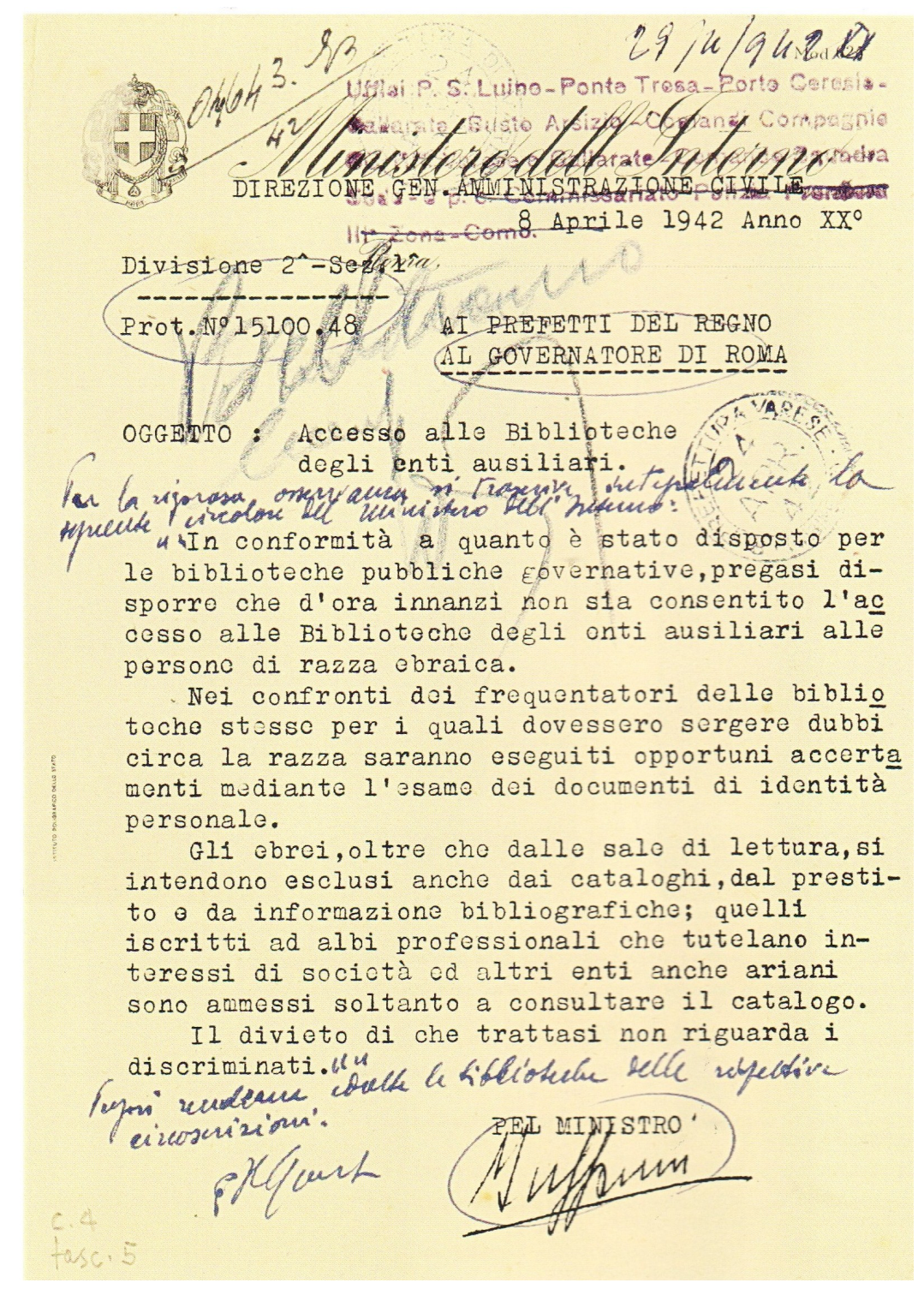

4. Divieto di accesso alle biblioteche. Archivio di Stato, Milano, in La storia, cit. p. 78. 


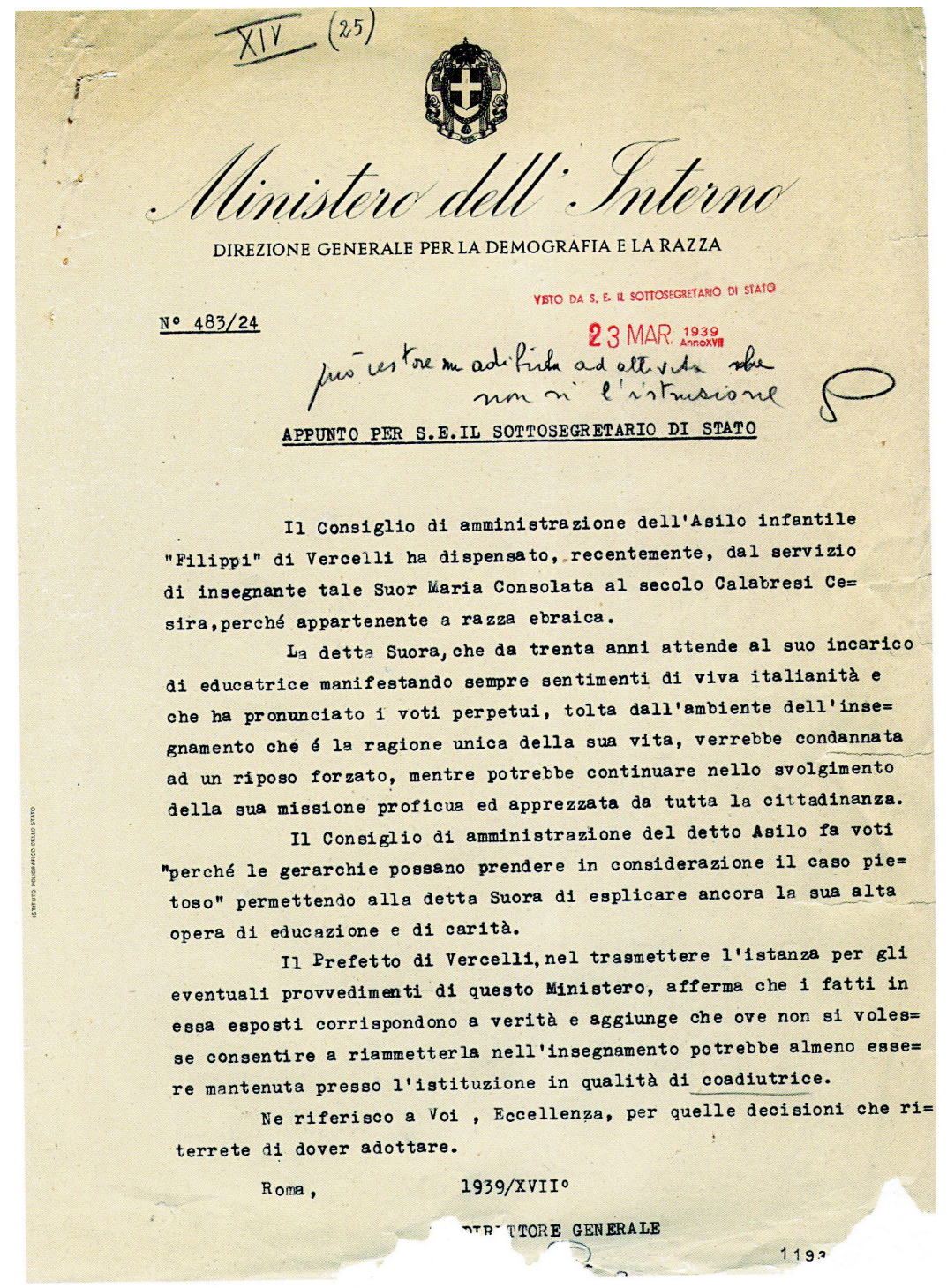

5. Il caso della suora insegnante di "razza ebraica". Archivio centrale dello Stato, Roma, in La storia, cit. p. 72 . 


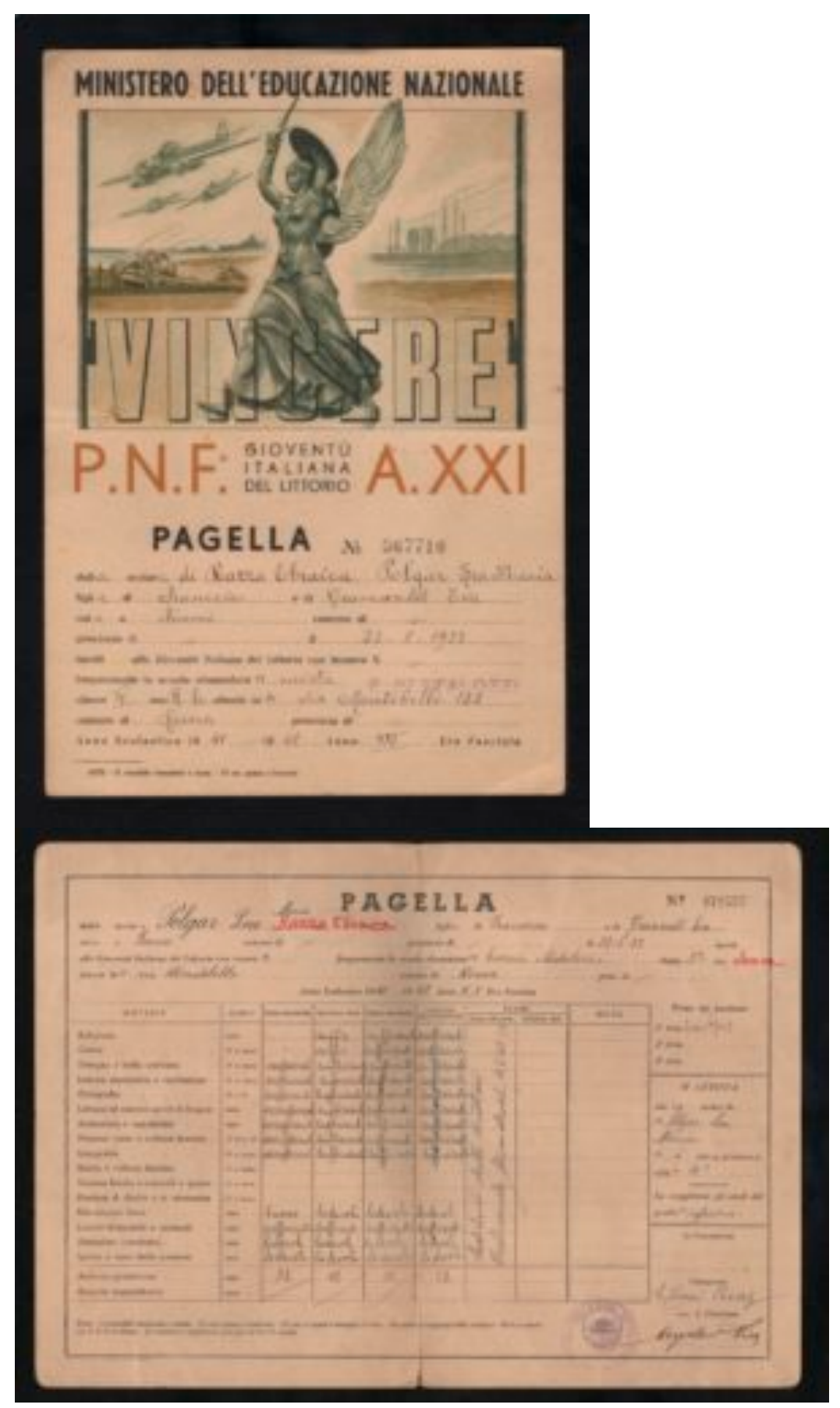

6. (fronte/retro): Pagella di Lea Polgar, bambina di «razza ebraica». Archivio privato della famiglia Polgar.

\section{Gli ebrei in Italia fino al 1938}

Da oltre duemila anni gli ebrei vivono nel territorio italiano. Nel corso di questo lungo periodo la storia della comunità ebraica, numericamente minore rispetto alla maggioranza della popolazione cristiana, è stata funestata da discriminazioni, ghettizzazioni ed espulsioni, fino al XIX secolo, 
quando, durante il Risorgimento, gli ebrei italiani raggiunsero la parità dei diritti ed entrarono a fare pienamente parte, come cittadini, dello Stato italiano appena costituito.

Agli inizi del XX secolo gli ebrei erano circa 45.000, corrispondenti all'uno per mille della popolazione della penisola; dopo l'emancipazione ottocentesca essi si erano integrati nella società e svolgevano qualsiasi tipo di professione, dalle più umili - molti lavoravano nel commercio ambulante, soprattutto in città come Roma, Livorno, Trieste - alle più elevate, essendo impiegati nell'industria, nelle banche e assicurazioni, nell'esercito, nel mondo della cultura e della pubblica amministrazione. Sidney Sonnino, Presidente del Consiglio nel primo decennio del Novecento, era ebreo da parte di padre; erano ebrei anche Ernesto Nathan, sindaco di Roma (1907-1913), Arnaldo Momigliano, storico dell'età antica, l'artista Amedeo Modigliani e molti altri personaggi italiani d'inizio secolo. Gli ebrei presero parte, come la restante popolazione, alla Prima guerra mondiale, e molti di loro furono decorati per il loro coraggio e patriottismo.

La progressiva secolarizzazione della società tra Ottocento e Novecento fu causa e conseguenza di un incremento dei matrimoni misti tra ebrei e cristiani, tanto che superarono la soglia del $30 \%$ all'inizio degli anni Trenta.

Con l'avvento del fascismo la situazione per gli ebrei non mutò. Come la maggior parte degli italiani essi aderirono regime che si andava consolidando nel Paese; d'altro canto, fino alla seconda metà degli anni Trenta, il fascismo non manifestò nessun sentimento antisemita. Alcuni ebrei indossarono la camicia nera con convinzione, ricoprendo anche ruoli importanti, come nel caso dell'imprenditore Guido Jung, Ministro delle Finanze nel Governo Mussolini dal 1932 al 1935. Altri invece si opposero subito al fascismo, pagando questa scelta con l'esilio, il confino o la vita stessa: i fratelli Carlo e Nello Rosselli, fondatori del giornale antifascista «Giustizia e libertà», rifugiati in Francia, furono lì raggiunti e uccisi da sicari fascisti nel 1937.

Le cose cambiarono dalla metà degli anni Trenta, come conseguenza della politica estera di Mussolini: parallelamente alla guerra in Etiopia e alla conquista dell'Impero (maggio 1936), si diffusero, ad opera della propaganda del regime, idee razziste contro le popolazioni nere, descritte come primitive e inferiori, mentre, con l'avvicinamento alla Germania antisemita di Hitler, attraverso il patto italo-tedesco («Asse Roma-Berlino», ottobre 1936), si sviluppò anche nell’Italia fascista una forte azione propagandistica antiebraica.

Nelle dichiarazioni dello stesso Mussolini ci fu una svolta radicale, nel giro di pochi anni: nel 1932, in un'intervista rilasciata al giornalista e scrittore Emil Ludwig, Mussolini affermava: «l'antisemitismo non esiste in Italia [...]. Gli ebrei italiani si sono comportati sempre bene come cittadini e come soldati si sono battuti coraggiosamente. Essi occupano posti elevati nelle Università, nell'esercito, nelle banche». Viceversa, nel settembre del 1938, in un discorso pubblico a Trieste, davanti a una folla oceanica, egli dichiarò che il problema razziale era di «scottante attualità» e che l'ebraismo era ormai diventato «un nemico irreconciliabile del fascismo». In questo stesso discorso, Mussolini sottolineava che il razzismo fascista non era conseguente a imitazioni o suggestioni, riferendosi evidentemente all'alleato nazista, ma era nato in Italia in seguito alla costituzione dell'Impero, come si evince dalle sue parole: «poiché la storia ci insegna che gli imperi si conquistano con le armi ma si tengono con il prestigio, occorre una chiara, severa coscienza razziale che stabilisca non soltanto delle differenze, ma delle superiorità nettissime».

Le «superiorità nettissime» della razza italiana «ariana» su quelle nera e semita, erano state presentate al popolo italiano già due mesi prima del discorso di Trieste, in un testo programmatico dal titolo Il fascismo e i problemi della razza. Questo documento, noto anche come Manifesto della Razza o Manifesto degli scienziati razzisti, fu pubblicato il 14 luglio 1938 nelle pagine centrali del 
«Giornale d'Italia», il quotidiano più letto di allora e, una seconda volta, nel primo numero della rivista La Difesa della Razza, ai primi di agosto dello stesso anno.

L'antiebraismo fascista era esploso, tra lo sgomento e l'incredulità degli ebrei italiani.

Il "Manifesto degli scienziati" razzisti era apparso incorniciato nel mezzo della prima pagina di un quotidiano con la violenza di un sasso o di un pugno che frantuma una vetrata.

«Esiste una razza italiana», gridava quel manifesto di fronte al mondo. "Gli ebrei non

appartengono alla razza italiana», e ancora altre parole infette: l'unica popolazione diversa sul suolo italiano risultava quella ebraica.

Le schegge della vetrata continuavano a cadere con moto incessante sulle sagome di ebrei che tentavano di ripararsi la testa con il braccio. Era come se ognuno di loro avesse custodito a lungo un segreto con se stesso e ora fosse stato smascherato.[1]

\section{Le leggi razziali del 1938}

Nell'introduzione al Manifesto della Razza si spiegava che un gruppo di scienziati, intellettuali e professori fascisti aveva scritto un documento per chiarire la posizione del regime nei confronti della questione razziale. Seguiva un discorso in dieci punti nel quale, oltre all'affermazione dell'esistenza di razze umane, alcune delle quali erano piccole e inferiori e altre grandi e superiori, trovavano spazio le teorie razziali basate su criteri biologici, per cui l'appartenenza alla razza ebraica o «ariana» non era data dalla fede religiosa, ma dalla discendenza di sangue; quindi poteva accadere che una persona figlia di ebrei, anche se convertita alla fede cattolica, comunque venisse perseguitata in quanto ebrea.

Nel mese di agosto venne costituito un ufficio del Ministero dell'Interno, «La Direzione generale per la demografia e la razza», noto come Demorazza, che schedò la popolazione ebraica, attraverso un censimento su basi razziste: 46.656 (37.341 italiani e 9.415 stranieri) si dichiarano ebrei; era 1'1,1 per mille della popolazione italiana, formata da quasi 44 milioni di abitanti. Questa minoranza fu perseguitata dalle leggi razziali fasciste, che vennero decise, scritte e divulgate nell'autunno del 1938.

Il Consiglio dei Ministri approvò, per volere di Mussolini, i primi decreti legge contro gli ebrei l'1 e il 2 settembre 1938; essi presero di mira il mondo della scuola e gli ebrei stranieri. Per essere certi di quali fossero le persone da colpire, il Gran Consiglio del Fascismo, nella seduta del 6 ottobre, stabilì i criteri per la definizione della «razza ebraica». L'intero corpo dei provvedimenti antiebraici venne poi approvato dal Consiglio dei Ministri in novembre, quando venne promulgato il Regio decreto legge n. 1728 del 17 novembre 1938: Provvedimenti per la difesa della razza italiana.

Da quel momento, fino al 1943, il regime emanò una miriade di provvedimenti, con lo scopo di attuare la normativa in modo capillare; si trattava di escludere gli ebrei da qualsiasi ambito della vita pubblica: dal lavoro alla scuola, dal mondo della cultura e dello sport, allo spettacolo.

Addirittura gli furono preclusi i luoghi di divertimento, come le spiagge pubbliche; essi, inoltre, non potevano possedere una radio; era loro vietata la possibilità di avere personale «ariano» alle proprie dipendenze, o, se medici, di avere pazienti non ebrei. Il razzismo si diffuse al punto che, come era già successo in altre parti d'Europa sotto l'influenza nazista (in Germania, Romania, Ungheria e Austria), gli ebrei fossero esclusi anche da bar, ristoranti e negozi. 
I provvedimenti contro gli ebrei continuavano a cadere a scansione lenta, come quei goccioloni radi ma già carichi che preludono alla tempesta. Si ritrovarono fradici senza neanche essersene accorti. Le Leggi diventarono operative ancor prima che fossero pubblicate.[2]

\section{Le leggi razziali nel mondo della scuola}

Dal Regio decreto del 5 settembre 1938, n. 1390, Provvedimenti per la difesa della razza nella scuola fascista:

Art. 1. All'ufficio di insegnante nelle scuole statali e parastatali, di qualsiasi ordine e grado [...] non potranno essere ammesse persone di razza ebraica.

Art. 2. Alle scuole di qualsiasi ordine e grado, ai cui studi sia riconosciuto effetto legale, non potranno essere iscritti alunni di razza ebraica.

Giuseppe Bottai, ministro dell'Educazione Nazionale dal 1936 al 1943, si occupò di regolamentare, con particolare perizia e accanimento, l'applicazione delle leggi razziali nei settori dell'istruzione e della cultura, tanto che firmò più di settecento provvedimenti, che uscirono tra il 1938 e il 1943 nella scuola e nell'Università; in essi si sottolineava l'esclusione dei cittadini di «razza ebraica» da qualsiasi ambito dell'istruzione e della formazione.

Settecento provvedimenti in cinque anni: in media due-tre volte la settimana il Ministero si premurò di diramare documenti attraverso i quali si ribadiva l'esclusione di questa minoranza dall'intero mondo dell'educazione, quindi dalle aule degli istituti di ogni ordine e grado, ma anche dai concorsi, dalle biblioteche, dalle segreterie e addirittura dai libri di testo, che non dovevano essere di autori ed editori ebrei.

Questa mole di documentazione è rimasta seppellita tra gli scaffali della biblioteca del Miur, in particolare nei Bollettini ufficiali del Ministero dell'Istruzione, che raccolgono tutte le disposizioni regolanti la vita della scuola italiana dal 1874 al 2012, fino a quando la bibliotecaria Vincenza Iossa e Manuele Gianfrancesco, dottore in storia moderna e contemporanea, con l'aiuto di alcuni studenti universitari, non hanno provveduto a spogliarne i contenuti, alla ricerca delle disposizioni relative all'allontanamento degli ebrei dalla scuola. Dice Vincenza Iossa, intervistata da RadioTre Suite il 27 gennaio 2020: «questa legge così dura, forte e intransigente, fu applicata e diffusa con il documento più debole: la circolare ministeriale».

Il risultato di tale studio capillare e minuzioso è sorprendente ha portato alla creazione di un volume, in formato cartaceo, ma anche divulgato a uso didattico in pdf sul sito https://www.scuolaememoria.it, che contiene, oltre alla parte introduttiva con interessanti considerazioni sul tema del rapporto tra regime fascista, razzismo di Stato e normativa scolastica, un'utile selezione di documenti originali che possono essere utilizzati per un percorso laboratoriale in classe, come suggerito in coda all'articolo.

Le circolari nei Bollettini del Miur testimoniano quanti e quali furono le persone colpite, a vario titolo, dai provvedimenti del 1938 nel mondo dell'istruzione: i presidi e i docenti delle scuole medie e superiori licenziati furono 279 e quasi un centinaio i professori delle università; i liberi docenti abilitati oltre 200; gli aiuti, assistenti e professori incaricati più di 130; i direttori e insegnanti di scuola elementare oltre 100. A tutti loro fu vietato insegnare. Tra di essi alcuni dei maggiori rappresentanti della cultura e della scienza nazionali, come i matematici Vito Volterra, Federigo Enriques, Guido Fubini; i fisici Bruno Rossi, Giulio Racah e il futuro premio Nobel Emilio Segrè; il 
chimico Mario Giacomo Levi; l'anatomista Giuseppe Levi; gli economisti Gustavo Del Vecchio e Gino Luzzato; il giurista Edoardo Volterra; gli storici Arnaldo Momigliano e Roberto Lopez; gli storici dell'arte Paolo D'Ancona e Doro Levi; gli storici della letteratura Attilio Momigliano ed Ezio Levi D'Ancona. Dovette abbandonare le sue ricerche in Italia l'allora trentenne Rita Levi Montalcini, insignita del premio Nobel per la medicina nel 1986, così come Salvatore Luria (premio Nobel per la medicina nel 1969) e Franco Modigliani (premio Nobel per l'Economia nel 1985).

Con le leggi razziali il regime non si limitò a escludere fisicamente le persone dalle aule della scuola pubblica, ma volle anche annullare la loro voce e il loro pensiero, conservato nelle pagine dei libri: furono più di cento i testi di autori, curatori ed editori ebrei che vennero banditi dal commercio ed eliminati dalle biblioteche; dai libri di fiabe ai testi scolastici di qualsiasi materia, fino alle carte geografiche e ai volumi universitari, un patrimonio culturale, che venne cancellato perché considerato una tremenda minaccia per la «purezza della razza». In questo modo il mondo dell'istruzione veniva depauperato enormemente essendo vietati anche i libri di autori «ariani» che facevano riferimenti ad autori ebrei morti dopo il 1850.

Oltre ai docenti espulsi vi fu un folto numero di professionisti che dovette abbandonare il proprio luogo di lavoro: il personale licenziato che lavorava in accademie e società scientifiche, letterarie, storiche e museali corrispondeva a più di 650 unità.

Ma la maggioranza degli esclusi dal mondo della cultura e della scuola è rappresentata da coloro cui fu vietato studiare, ovvero dalle migliaia di bambini e ragazzi che, senza nessun preavviso, furono costretti a lasciare il loro banco vuoto e che provarono in quel momento un penoso isolamento. Come emerge nella testimonianza di Piero Terracina:

Era martedi 15 novembre 1938. Io entrai in classe, come sempre; ebbi l'impressione che i miei compagni di classe mi guardassero con un'aria un po'strana, non quella consueta, ma non ci feci molto caso. La mia insegnante, che tra l'altro portavo dalla prima elementare, era stata sempre lei, dalla prima alla quinta, mi era sempre sembrato che mi volesse bene; qualche volta mi mandava nelle classi superiori o per recitare una poesia o per fare leggere un compito, e quindi era un rapporto direi molto buono; non era di religione ebraica, anzi tutta la famiglia era fascista della prima ora. E insomma la mia insegnante quel giorno fece l'appello, ma non chiamò il mio nome. Mi ricordo che soltanto alla fine mi disse: "Terracina esci». Rimasi un po' meravigliato, anche se in casa avevo inteso qualche cosa, ma non ci avevo fatto caso perché non erano cose per bambini, erano cose da grandi. E io risposi subito: «Perché, che cosa ho fatto?». E lei disse: «Devi uscire perché sei ebreo». Mi disse queste parole, secche secche, senza aggiungere assolutamente altro, $e$ io dovetti uscire. In quel momento ebbi la sensazione che il mondo mi crollasse addosso. Io ero stato educato all'amore per lo studio, per la scuola. Mia madre non tralasciava occasione per ricordare che se uno fosse riuscito nello studio, poi nella vita sarebbe stato tutto molto più facile. E quindi quando mi disse: "Tu non puoi stare qui, non puoi stare a scuola, non puoi studiare» ebbi veramente una sensazione terribile, perché pensai subito: "E che cosa farò mai nella vita?».[3]

\section{Conclusioni}

Le leggi antiebraiche del novembre del 1938 esclusero migliaia di persone dal settore della cultura e dalla scuola pubblica. Le comunità ebraiche, cui fu permesso di occuparsi degli alunni e dei docenti espulsi, dopo un primo smarrimento iniziale, organizzarono, dove fu possibile, dei corsi scolastici paralleli, in case private o locali annessi alle sinagoghe; in alcune città, tuttavia, vennero istituite delle classi separate all'interno delle stesse scuole pubbliche: le sezioni speciali con studenti e 
insegnanti ebrei. Esse restarono vive fino all'anno scolastico 1942-43; con l'occupazione nazifascista, infatti, le sezioni speciali ebraiche sparirono: a chi, dal 1938, era stato vietato insegnare e studiare, dall'autunno del 1943, fu vietato vivere. Molti di loro riuscirono a nascondersi e si salvarono, ma moltissimi furono scoperti, deportati e assassinati nei campi di sterminio nazisti. A noi il compito di non dimenticarli, di riscoprire la loro voce e le loro storie e, parallelamente, attraverso laboratori come questo, ridisegnare il percorso che portò a svuotare migliaia di banchi e di cattedre. In questo modo, fare memoria del passato serve da monito e prevenzione, perché, come afferma Michele Sarfatti, storico della Shoah, nella Prefazione del volume Vietato studiare, vietato insegnare, «la scuola odierna deve rimanere un baluardo nella difesa dei diritti di tutte le minoranze e nel respingimento dell'antisemitismo e di qualsiasi razzismo»[4].

\section{Bibliografia}

- S. Berger e M. Pezzetti (a cura di), 1938. La storia, Gangemi Editore, 2017.

- V. Iossa e M. Gianfrancesco (a cura di), Vietato studiare vietato insegnare. Il Ministero dell'educazione nazionale e l'attuazione delle norme antiebraiche 1938-1943, Palombi editori, 2019.

- L. Levi, Questa sera è già domani, E/O, 2018.

\section{Filmografia}

- P. Garribba (a cura di), Piero Terracina, un ebreo romano, Aned-Roma, 2000.

- P. Suber, 1938. Quando scoprimmo di non essere più italiani, film documentario, Gedi Gruppo editoriale, 2018.

Note:

[1] L. Levi, Questa sera è già domani, E/O, 2018, pp. 60-61.

[2] L. Levi, Questa sera è già domani, E/O, 2018, p. 63

[3] Piero Terracina, un ebreo romano, videointervista a cura dell'Aned di Roma, 2000.

[4] Vietato studiare vietato insegnare. Il Ministero dell'educazione nazionale e l'attuazione delle norme antiebraiche 1938-1943, cit., p. 15. 